\title{
FUNDAMENTAL PRINCIPLES
}

Convective heat transfer, or simply, convection, is the study of heat transport processes effected by the flow of fluids. The very word convection has its roots in the Latin verbs convecto-are and convěho-věherre [1],* which mean to bring together or to carry into one place [2]. Convective heat transfer has grown to the status of a contemporary science because of people's desire to understand and predict how a fluid flow will act as a "carrier" or "conveyor belt" for energy and matter.

Convective heat transfer is clearly, a field at the interface between two older fields: heat transfer and fluid mechanics. For this reason, the study of any convective heat transfer problem must rest on a solid understanding of basic heat transfer and fluid mechanics principles. The objective in this chapter is to review these principles in order to establish a common language to debate the more specific issues addressed in later chapters.

Before reviewing the foundation of convective heat transfer methodology, it is worth reexamining the historic relationship between fluid mechanics and heat transfer at the interface we call convection. Especially during the past 100 years, heat transfer and fluid mechanics have enjoyed a symbiotic relationship in their development, a relationship where one field was stimulated by the curiosity in the other field. Examples of this symbiosis abound in the history of boundary layer theory and natural convection. The field of convection heat transfer grew out of this symbiosis, and if we are to learn anything from history, important advances in convection will continue to result from this symbiosis. Thus, the student and the future researcher would be well advised to devote equal attention to fluid mechanics and heat transfer literature.

* Numbers in brackets indicate references at the end of each chapter. 


\subsection{MASS CONSERVATION}

The first principle to review is undoubtedly the oldest: It is the conservation of mass in a closed system or the "continuity" of mass through a flow (open) system. From engineering thermodynamics, we recall the mass conservation statement for a control volume [3]:

$$
\frac{\partial M_{\mathrm{cv}}}{\partial t}=\sum_{\substack{\text { inlet } \\ \text { ports }}} \dot{m}-\sum_{\substack{\text { outlet } \\ \text { ports }}} \dot{m}
$$

where $M_{\mathrm{cv}}$ is the mass instantaneously trapped inside the control volume (cv), while the $\dot{m}$ 's are the mass flow rates associated with flow into and out of the control volume. In convective heat transfer, we are usually interested in the velocity and temperature distribution in a flow region near a solid wall; hence, the control volume to consider is the infinitesimally small $\Delta x \Delta y$ box drawn around a fixed location $(x, y)$ in a flow field. In Fig. 1.1, as in most of the problems analyzed in this book, the flow field is two-dimensional (i.e., the same in any plane parallel to the plane of Fig. 1.1); in a three-dimensional flow field, the control volume of interest would be the parallelepiped $\Delta x \Delta y \Delta z$. Taking $u$ and $v$ as the local velocity components at point $(x, y)$, the mass conservation equation (1.1) requires that

$$
\begin{aligned}
\frac{\partial}{\partial t}(\rho \Delta x \Delta y)= & \rho u \Delta y+\rho v \Delta x-\left[\rho u+\frac{\partial(\rho u)}{\partial x} \Delta x\right] \Delta y \\
& -\left[\rho v+\frac{\partial(\rho v)}{\partial y} \Delta y\right] \Delta x
\end{aligned}
$$

or, dividing through by the constant size of the control volume $(\Delta x \Delta y)$,

$$
\frac{\partial \rho}{\partial t}+\frac{\partial(\rho u)}{\partial x}+\frac{\partial(\rho v)}{\partial y}=0
$$

In a three-dimensional flow, an analogous argument yields

$$
\frac{\partial \rho}{\partial t}+\frac{\partial(\rho u)}{\partial x}+\frac{\partial(\rho v)}{\partial y}+\frac{\partial(\rho w)}{\partial z}=0
$$

where $w$ is the velocity component in the $z$ direction. The local mass conservation statement (1.4) can also be written as 


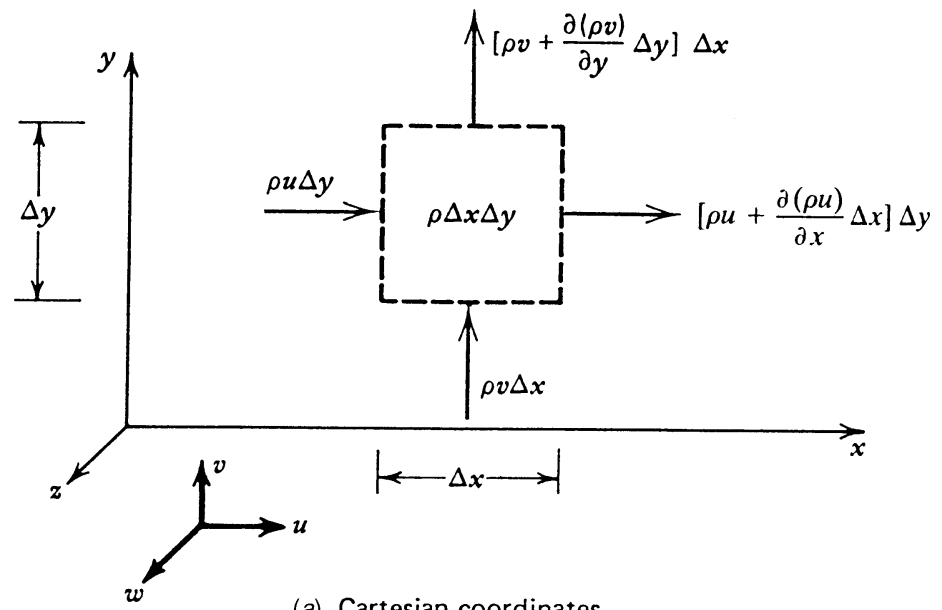

(a) Cartesian coordinates

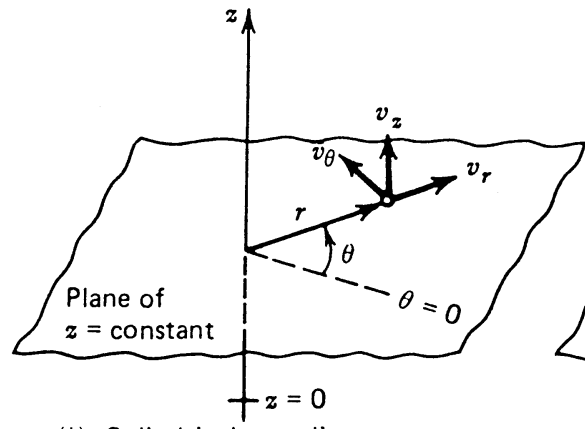

(b) Cylindrical coordinates

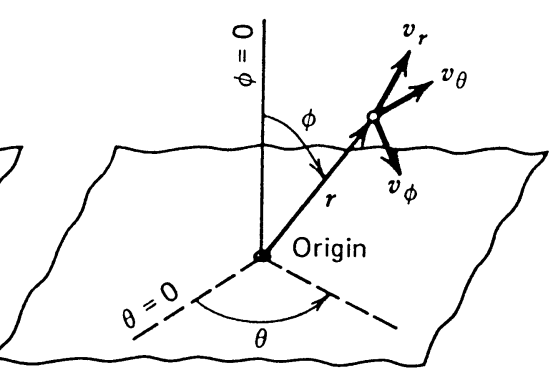

(c) Spherical coordinates

Figure 1.1 Mass conservation and systems of coordinates.

$$
\frac{\partial \rho}{\partial t}+u \frac{\partial \rho}{\partial x}+v \frac{\partial \rho}{\partial y}+w \frac{\partial \rho}{\partial z}+\rho\left(\frac{\partial u}{\partial x}+\frac{\partial v}{\partial y}+\frac{\partial w}{\partial z}\right)=0
$$

or

$$
\frac{D \rho}{D t}+\rho \nabla \cdot \mathbf{v}=0
$$

In expression (1.6), $\mathbf{v}$ is the velocity vector $(u, v, w)$, and $D / D t$ represents the "material derivative" operator, encountered frequently in convective heat and mass transfer, 


$$
\frac{D}{D t}=\frac{\partial}{\partial t}+u \frac{\partial}{\partial x}+v \frac{\partial}{\partial y}+w \frac{\partial}{\partial z}
$$

Of particular interest to classroom treatment of the convection problem is the wide class of flows in which temporal and spatial variations in density are negligible relative to the local variations in velocity. For this class, the mass conservation statement reads

$$
\frac{\partial u}{\partial x}+\frac{\partial v}{\partial y}+\frac{\partial w}{\partial z}=0
$$

The equivalent forms of eq. (1.8) in cylindrical and spherical coordinates are (Fig. 1.1)

$$
\frac{\partial v_{r}}{\partial r}+\frac{v_{r}}{r}+\frac{1}{r} \frac{\partial v_{\theta}}{\partial \theta}+\frac{\partial v_{z}}{\partial z}=0
$$

and

$$
\frac{1}{r} \frac{\partial}{\partial r}\left(r^{2} v_{r}\right)+\frac{1}{\sin \phi} \frac{\partial}{\partial \phi}\left(v_{\phi} \sin \phi\right)+\frac{1}{\sin \phi} \frac{\partial v_{\theta}}{\partial \theta}=0
$$

It is tempting to regard eqs. (1.8) - (1.10) as valid only for incompressible fluids; in fact, their derivation shows that they apply to flows (not fluids) where the density and velocity gradients are such that the $D \rho / D t$ terms are negligible relative to the $\rho \nabla \cdot \mathbf{v}$ terms in eq. (1.6). Most of the gas flows encountered in heat exchangers, heated enclosures, and porous media obey the simplified version of the mass conservation principle [eqs. (1.8)-(1.10)].

\subsection{FORCE BALANCES (MOMENTUM EQUATIONS)}

From the dynamics of thrust or propulsion systems, we recall that the instantaneous force balance on a control volume requires that [3]

$$
\frac{\partial}{\partial t}\left(M v_{n}\right)_{\mathrm{cv}}=\sum F_{n}+\sum_{\substack{\text { inlet } \\ \text { ports }}} \dot{m} v_{n}-\sum_{\substack{\text { outlet } \\ \text { ports }}} \dot{m} v_{n}
$$

where $n$ is the direction chosen for analysis and $v_{n}$ and $F_{n}$ are the projections of fluid velocity and forces in the $n$ direction. Equation (1.11) is recognized in the literature as the momentum principle or momentum theorem: In essence, eq. (1.11) is the control volume formulation of Newton's second law of motion, where in addition to terms accounting for forces and mass $\times$ acceleration, we now have the impact due to the flow of momentum into the control volume, 
plus the reaction associated with the flow of momentum out of the control volume. In the two-dimensional flow situation of Fig. 1.2, we can write two force balances of type (1.11), one for the $x$ direction and the other for the $y$ direction.

Consider now the special form taken by eq. (1.11) when applied to the finitesize control volume $\Delta x \Delta y$ drawn around point $(x, y)$ in Fig. 1.2. Consider first the balance of forces in the $x$ direction. In Fig. 1.2a, showing the $\Delta x \Delta y$ control volume, we see the sense of the impact and reaction forces associated with the flow of momentum through the control volume. In Fig. $1.2 b$, we see the more classical forces represented by the normal stress $\left(\sigma_{x}\right)$, tangential stress $\left(\tau_{x y}\right)$, and the $x$-direction body force per unit volume $(X)$.

Projecting all these forces on the $x$ axis, we obtain

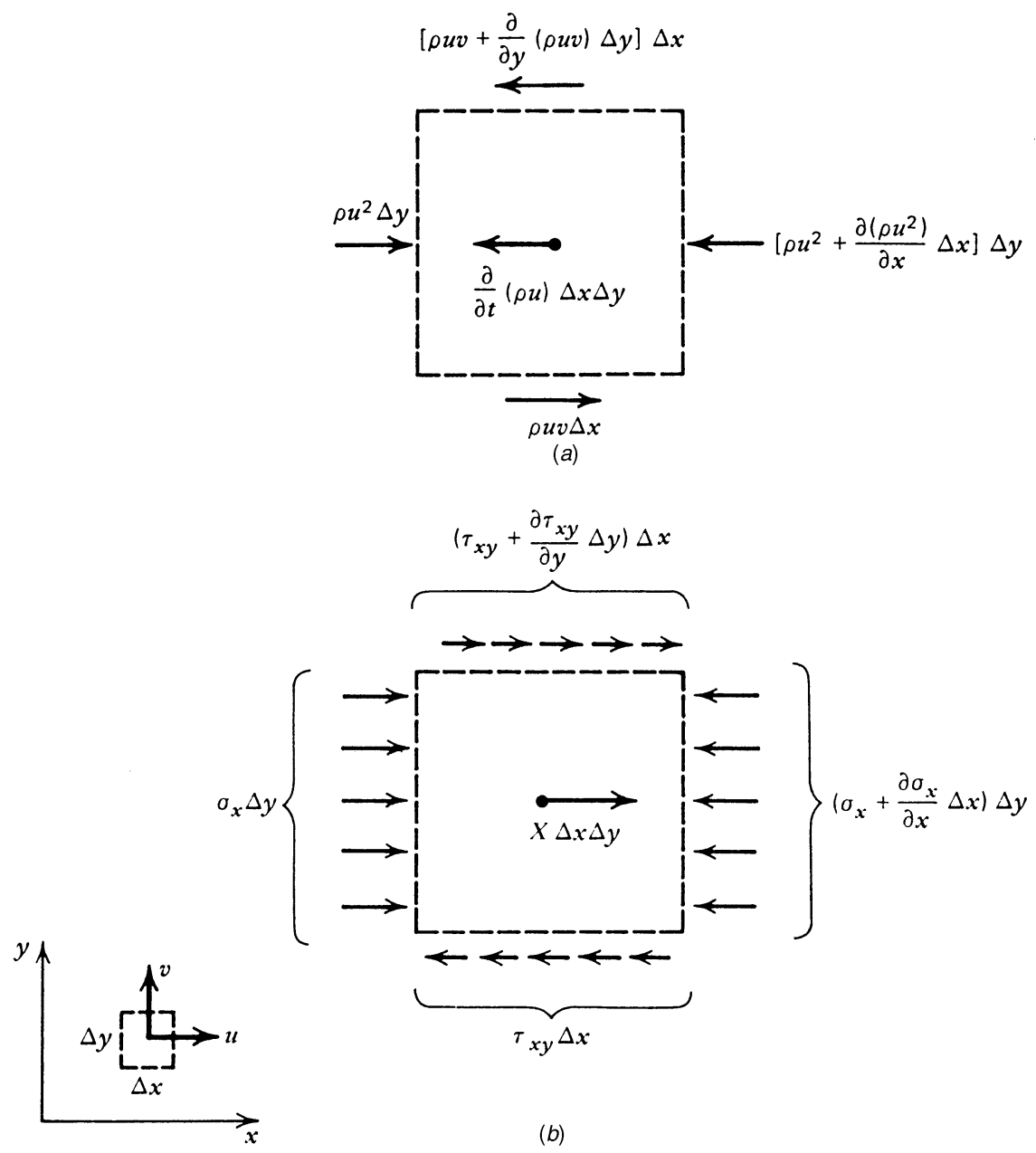

Figure 1.2 Force balance in the $x$ direction on a control volume in two-dimensional flow. 


$$
\begin{aligned}
& -\frac{\partial}{\partial t}(\rho u \Delta x \Delta y)+\rho u^{2} \Delta y-\left[\rho u^{2}+\frac{\partial}{\partial x}\left(\rho u^{2}\right) \Delta x\right] \Delta y \\
& \quad+\rho u v \Delta x-\left[\rho u v+\frac{\partial}{\partial y}(\rho u v) \Delta y\right] \Delta x \\
& \quad+\sigma_{x} \Delta y-\left(\sigma_{x}+\frac{\partial \sigma_{x}}{\partial x} \Delta x\right) \Delta y-\tau_{x y} \Delta x \\
& \quad+\left(\tau_{x y}+\frac{\partial \tau_{x y}}{\partial y} \Delta y\right) \Delta x+X \Delta x \Delta y=0
\end{aligned}
$$

or, dividing by $\Delta x \Delta y$ in the limit $(\Delta x, \Delta y) \rightarrow 0$,

$$
\rho \frac{D u}{D t}+u\left[\frac{D \rho}{D t}+\rho\left(\frac{\partial u}{\partial x}+\frac{\partial v}{\partial y}\right)\right]=-\frac{\partial \sigma_{x}}{\partial x}+\frac{\partial \tau_{x y}}{\partial y}+X
$$

According to the mass conservation equation (1.6), the quantity in brackets is equal to zero; hence,

$$
\rho \frac{D u}{D t}=-\frac{\partial \sigma_{x}}{\partial x}+\frac{\partial \tau_{x y}}{\partial y}+X
$$

Next, we relate the stresses $\sigma_{x}$ and $\tau_{x y}$ to the local flow field by recalling the constitutive relations [4]

$$
\begin{aligned}
& \sigma_{x}=P-2 \mu \frac{\partial u}{\partial x}+\frac{2}{3} \mu\left(\frac{\partial u}{\partial x}+\frac{\partial v}{\partial y}\right) \\
& \tau_{x y}=\mu\left(\frac{\partial u}{\partial y}+\frac{\partial v}{\partial x}\right)
\end{aligned}
$$

These relations are of empirical origin: They summarize the experimental observation that a fluid packet offers no resistance to a change of shape, but resists the time rate of a change of shape. Equations (1.15) and (1.16) define the measurable coefficient of viscosity $\mu$. Combining eqs. (1.14)-(1.16) yields the Navier-Stokes equation,

$$
\begin{aligned}
\rho \frac{D u}{D t}= & -\frac{\partial P}{\partial x}+\frac{\partial}{\partial x}\left[2 \mu \frac{\partial u}{\partial x}-\frac{2 \mu}{3}\left(\frac{\partial u}{\partial x}+\frac{\partial v}{\partial y}\right)\right] \\
& +\frac{\partial}{\partial y}\left[\mu\left(\frac{\partial u}{\partial y}+\frac{\partial v}{\partial x}\right)\right]+X
\end{aligned}
$$


Of particular interest is the case when the flow may be treated as incompressible and the viscosity $\mu$ may be regarded as constant. Then the $x$ momentum equation reduces to

$$
\rho\left(\frac{\partial u}{\partial t}+u \frac{\partial u}{\partial x}+v \frac{\partial u}{\partial y}\right)=-\frac{\partial P}{\partial x}+\mu\left(\frac{\partial^{2} u}{\partial x^{2}}+\frac{\partial^{2} u}{\partial y^{2}}\right)+X
$$

A similar equation can be derived from the force balance in the $y$ direction. For a three-dimensional flow in the $(x, y, z),(u, v, w)$ Cartesian system, the three momentum equations for $\rho, \mu \cong$ constant flows are

$$
\begin{array}{r}
\rho\left(\frac{\partial u}{\partial t}+u \frac{\partial u}{\partial x}+v \frac{\partial u}{\partial y}+w \frac{\partial u}{\partial z}\right) \\
=-\frac{\partial P}{\partial x}+\mu\left(\frac{\partial^{2} u}{\partial x^{2}}+\frac{\partial^{2} u}{\partial y^{2}}+\frac{\partial^{2} u}{\partial z^{2}}\right)+X \\
\rho\left(\frac{\partial v}{\partial t}+u \frac{\partial v}{\partial x}+v \frac{\partial v}{\partial y}+w \frac{\partial v}{\partial z}\right) \\
=-\frac{\partial P}{\partial y}+\mu\left(\frac{\partial^{2} v}{\partial x^{2}}+\frac{\partial^{2} v}{\partial y^{2}}+\frac{\partial^{2} v}{\partial z^{2}}\right)+Y \\
\rho\left(\frac{\partial w}{\partial t}+u \frac{\partial w}{\partial x}+v \frac{\partial w}{\partial y}+w \frac{\partial w}{\partial z}\right) \\
=-\frac{\partial P}{\partial z}+\mu\left(\frac{\partial^{2} w}{\partial x^{2}}+\frac{\partial^{2} w}{\partial y^{2}}+\frac{\partial^{2} w}{\partial z^{2}}\right)+Z
\end{array}
$$

Alternative forms of eqs. (1.19) are:

Vectorial notation:

$$
\rho \frac{D \mathbf{v}}{D t}=-\nabla P+\mu \nabla^{2} \mathbf{v}+\mathbf{F}
$$

where $\mathbf{F}$ is the body force vector per unit volume $(X, Y, Z)$.

Cylindrical coordinates (Fig. 1.1b):

$$
\begin{aligned}
& \rho\left(\frac{\partial v_{r}}{\partial t}+v_{r} \frac{\partial v_{r}}{\partial r}+\frac{v_{\theta}}{r} \frac{\partial v_{r}}{\partial \theta}-\frac{v_{\theta}^{2}}{r}+v_{z} \frac{\partial v_{r}}{\partial z}\right) \\
& =-\frac{\partial P}{\partial r}+\mu\left(\frac{\partial^{2} v_{r}}{\partial r^{2}}+\frac{1}{r} \frac{\partial v_{r}}{\partial r}-\frac{v_{r}}{r^{2}}+\frac{1}{r^{2}} \frac{\partial^{2} v_{r}}{\partial \theta^{2}}-\frac{2}{r^{2}} \frac{\partial v_{\theta}}{\partial \theta}+\frac{\partial^{2} v_{r}}{\partial z^{2}}\right)+F_{r}
\end{aligned}
$$




$$
\begin{gathered}
\rho\left(\frac{\partial v_{\theta}}{\partial t}+v_{r} \frac{\partial v_{\theta}}{\partial r}+\frac{v_{\theta}}{r} \frac{\partial v_{\theta}}{\partial \theta}+\frac{v_{r} v_{\theta}}{r}+v_{z} \frac{\partial v_{\theta}}{\partial z}\right) \\
=-\frac{1}{r} \frac{\partial P}{\partial \theta}+\mu\left(\frac{\partial^{2} v_{\theta}}{\partial r^{2}}+\frac{1}{r} \frac{\partial v_{\theta}}{\partial r}-\frac{v_{\theta}}{r^{2}}+\frac{1}{r^{2}} \frac{\partial^{2} v_{\theta}}{\partial \theta^{2}}+\frac{2}{r^{2}} \frac{\partial v_{r}}{\partial \theta}+\frac{\partial^{2} v_{\theta}}{\partial z^{2}}\right)+F_{\theta} \\
\rho\left(\frac{\partial v_{z}}{\partial t}+v_{r} \frac{\partial v_{z}}{\partial r}+\frac{v_{\theta}}{r} \frac{\partial v_{z}}{\partial \theta}+v_{z} \frac{\partial v_{z}}{\partial z}\right) \\
=-\frac{\partial P}{\partial z}+\mu\left(\frac{\partial^{2} v_{z}}{\partial r^{2}}+\frac{1}{r} \frac{\partial v_{z}}{\partial r}+\frac{1}{r^{2}} \frac{\partial^{2} v_{z}}{\partial \theta^{2}}+\frac{\partial^{2} v_{z}}{\partial z^{2}}\right)+F_{z}
\end{gathered}
$$

where $\left(v_{r}, v_{\theta}, v_{z}\right)$ and $\left(F_{r}, F_{\theta}, F_{z}\right)$ are the velocity and body force vectors.

Spherical coordinates (Fig. 1.1c):

$$
\begin{aligned}
& \rho\left(\frac{D v_{r}}{D t}-\frac{v_{\phi}^{2}+v_{\theta}^{2}}{r}\right) \\
& \quad=-\frac{\partial P}{\partial r}+\mu\left(\nabla^{2} v_{r}-\frac{2 v_{r}}{r^{2}}-\frac{2}{r^{2}} \frac{\partial v_{\phi}}{\partial \phi}-\frac{2 v_{\phi} \cot \phi}{r^{2}}-\frac{2}{r^{2} \sin \phi} \frac{\partial v_{\theta}}{\partial \theta}\right)+F_{r}
\end{aligned}
$$$$
\begin{aligned}
& \rho\left(\frac{D v_{\phi}}{D t}+\frac{v_{r} v_{\phi}}{r}-\frac{v_{\theta}^{2} \cot \phi}{r}\right) \\
& \quad=-\frac{1}{r} \frac{\partial P}{\partial \phi}+\mu\left(\nabla^{2} v_{\phi}+\frac{2}{r^{2}} \frac{\partial v_{r}}{\partial \phi}-\frac{v_{\phi}}{r^{2} \sin ^{2} \phi}-\frac{2 \cos \phi}{r^{2} \sin ^{2} \phi} \frac{\partial v_{\theta}}{\partial \theta}\right)+F_{\phi}
\end{aligned}
$$

$$
\begin{aligned}
\rho\left(\frac{D v_{\theta}}{D t}+\frac{v_{\theta} v_{r}}{r}+\frac{v_{\phi} v_{\theta} \cot \phi}{r}\right) \\
=-\frac{1}{r \sin \phi} \frac{\partial P}{\partial \theta}+\mu\left(\nabla^{2} v_{\theta}-\frac{v_{\theta}}{r^{2} \sin ^{2} \phi}+\frac{2}{r^{2} \sin \phi} \frac{\partial v_{r}}{\partial \theta}\right. \\
\left.\quad+\frac{2 \cos \phi}{r^{2} \sin ^{2} \phi} \frac{2 v_{\phi}}{\partial \theta}\right)+F_{\theta}
\end{aligned}
$$

where $\left(v_{r}, v_{\phi}, v_{\theta}\right)$ and $\left(F_{r}, F_{\phi}, F_{\theta}\right)$ are the velocity and body force vectors, and

$$
\frac{D}{D t}=\frac{\partial}{\partial t}+v_{r} \frac{\partial}{\partial r}+\frac{v_{\phi}}{r} \frac{\partial}{\partial \phi}+\frac{v_{\theta}}{r \sin \phi} \frac{\partial}{\partial \theta}
$$




$$
\nabla^{2}=\frac{1}{r^{2}} \frac{\partial}{\partial r}\left(r^{2} \frac{\partial}{\partial r}\right)+\frac{1}{r^{2} \sin \phi} \frac{\partial}{\partial \phi}\left(\sin \phi \frac{\partial}{\partial \phi}\right)+\frac{1}{r^{2} \sin ^{2} \phi} \frac{\partial^{2}}{\partial \theta^{2}}
$$

are the material derivative and Laplacian operators in spherical coordinates.

\subsection{FIRST LAW OF THERMODYNAMICS}

The preceding two principles-mass conservation and force balance-are in many cases sufficient for solving the flow part of the convective heat transfer

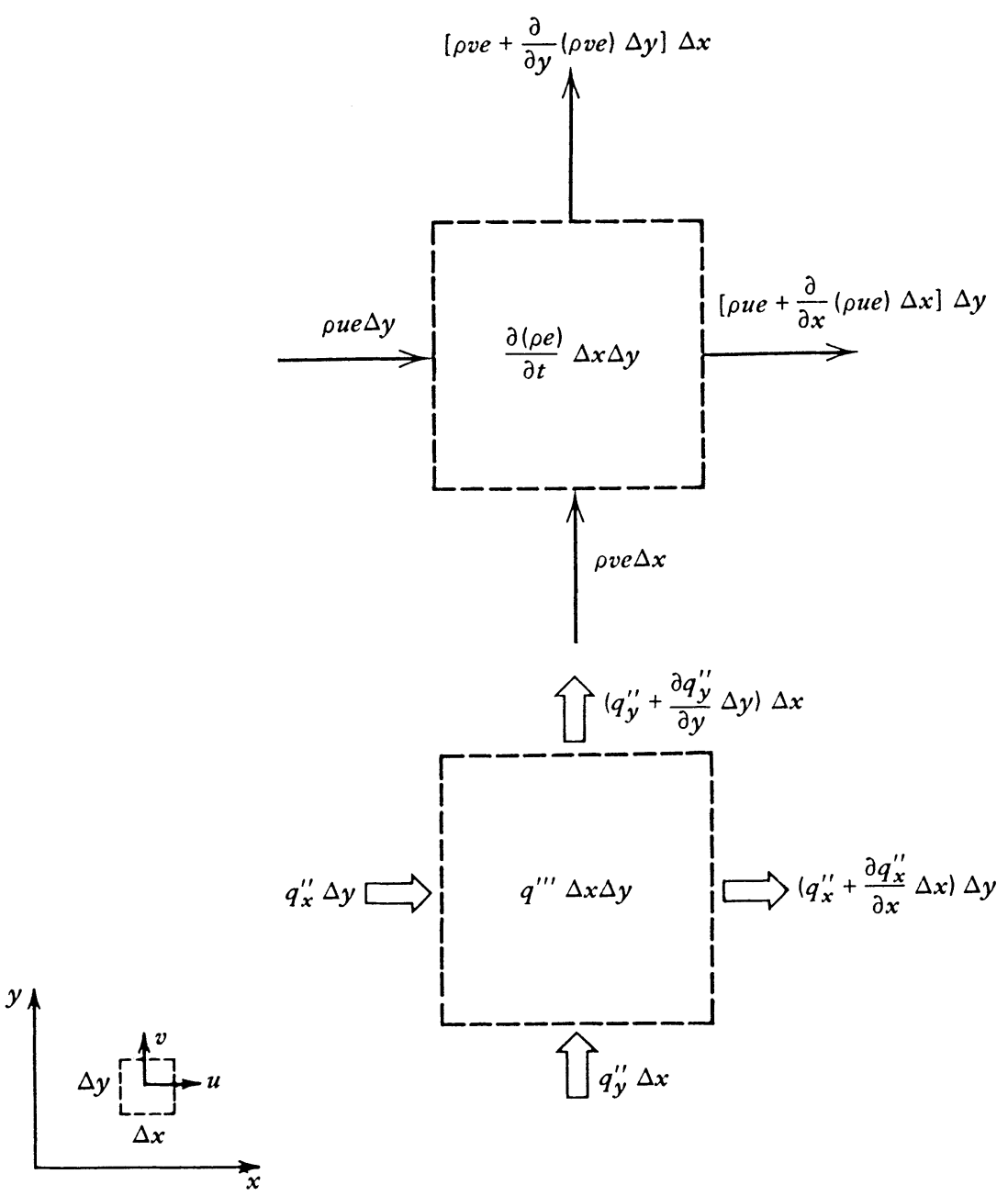

Figure 1.3 First law of thermodynamics applied to a control volume in two-dimensional flow (for work transfer, see Fig. 1.2). 
problem; note at this juncture the availability of four equations (mass conservation plus three force balances) for determining four unknowns (three velocity components plus pressure). The exception to this statement is the subject of Chapter 4, where the natural flow is driven by the heat administered to the flowing fluid. In all cases, however, the heat transfer part of the convection problem requires a solution for the temperature distribution through the flow, especially in the close vicinity of the solid walls bathed by the heat-carrying fluid stream (Chapter 2). The additional equation for accomplishing this ultimate objective is the first law of thermodynamics or the energy equation.

For the control volume of finite size $\Delta x \Delta y$ in Fig. 1.3, the first law of thermodynamics requires that

$$
\begin{aligned}
& \left(\begin{array}{l}
\text { rate of energy } \\
\text { accumulation in the } \\
\text { control volume }
\end{array}\right) \\
& =\left(\begin{array}{l}
\text { net transfer of } \\
\text { energy by fluid flow }
\end{array}\right)_{2}+\left(\begin{array}{l}
\text { net heat transfer } \\
\text { by conduction }
\end{array}\right)_{3} \\
& +\left(\begin{array}{l}
\text { rate of internal } \\
\text { heat generation (e.g., } \\
\text { electrical power } \\
\text { dissipation) }
\end{array}\right)_{4}-\left(\begin{array}{l}
\text { net work transfer } \\
\text { from the control } \\
\text { volume to its } \\
\text { environment }
\end{array}\right)_{5}
\end{aligned}
$$

According to the energy flow diagrams sketched in Fig. 1.3, the groups of terms above are

$$
\begin{aligned}
\{\cdot\}_{1}= & \Delta x \Delta y \frac{\partial}{\partial t}(\rho e) \\
\{\cdot\}_{2}= & -(\Delta x \Delta y)\left[\frac{\partial}{\partial x}(\rho u e)+\frac{\partial}{\partial y}(\rho v e)\right] \\
\{\cdot\}_{3}= & -(\Delta x \Delta y)\left(\frac{\partial q_{x}^{\prime \prime}}{\partial x}+\frac{\partial q_{y}^{\prime \prime}}{\partial y}\right) \\
\{\cdot\}_{4}= & (\Delta x \Delta y) q^{\prime \prime \prime} \\
\{\cdot\}_{5}= & (\Delta x \Delta y)\left(\sigma_{x} \frac{\partial u}{\partial x}-\tau_{x y} \frac{\partial u}{\partial y}+\sigma_{y} \frac{\partial v}{\partial y}-\tau_{y x} \frac{\partial v}{\partial x}\right) \\
& +(\Delta x \Delta y)\left(u \frac{\partial \sigma_{x}}{\partial x}-u \frac{\partial \tau_{x y}}{\partial y}+v \frac{\partial \sigma_{y}}{\partial y}-v \frac{\partial \tau_{y x}}{\partial x}\right)_{*}
\end{aligned}
$$


where $e, q_{x}^{\prime \prime}, q_{y}^{\prime \prime}$, and $q^{\prime \prime \prime}$ are the specific internal energy, heat flux in the $x$ direction, heat flux in the $y$ direction, and dissipation rate or rate of internal heat generation.

The origin of the dissipation rate term $\{\cdot\}_{5}$ lies in the work transfer effected by the normal and tangential stresses sketched in Fig. 1.2b. For example, the work done per unit time by the normal stresses $\sigma_{x}$ on the left side of the $\Delta x$ $\Delta y$ element is negative and equal to the force acting on the boundary $\left(\sigma_{x} \Delta y\right)$ times the boundary displacement per unit time $(u)$, which yields $-u \sigma_{x} \Delta y$. Similarly, the work transfer rate associated with normal stresses acting on the right side of the element is positive and equal to $\left[\sigma_{x}+\left(\partial \sigma_{x} / \partial x\right) \Delta x\right][u+$ $(\partial u / \partial x) \Delta x] \Delta y$. The net work transfer rate due to these two contributions is $\left[\sigma_{x}(\partial u / \partial x)+u\left(\partial \sigma_{x} / \partial x\right)\right](\Delta x \Delta y)$, as shown in the $\{\cdot\}_{5}$ term of eq. $\left(1.25^{\prime}\right)$.

Three more work transfer rates can be calculated in the same manner by examining the effect of the remaining three stresses, $\tau_{x y}$ in the $x$ direction and $\sigma_{y}$ and $\tau_{y x}$ in the $y$ direction. In the $\{\cdot\}_{5}$ expression above, the eight terms have been separated into two groups. It can be shown that the group denoted as $(\cdot)_{*}$ reduces to $-\rho(D / D t)\left(u^{2}+v^{2}\right) / 2$, which represents the change in kinetic energy of the fluid packet; in the present treatment, this change is considered negligible relative to the internal energy change $\partial(\rho e) / \partial t$ appearing in $\{\cdot\}_{1}$.

Assembling expressions (1.25) into the energy conservation statement that preceded them, and using constitutive relations (1.15) and (1.16), we obtain

$$
\rho \frac{D e}{D t}+e\left(\frac{D \rho}{D t}+\rho \nabla \cdot \mathbf{v}\right)=-\nabla \cdot \mathbf{q}^{\prime \prime}+q^{\prime \prime \prime}-P \nabla \cdot \mathbf{v}+\mu \Phi
$$

where $\mathbf{q}^{\prime \prime}$ is the heat flux vector $\left(q_{x}^{\prime \prime}, q_{y}^{\prime \prime}\right)$ and $\Phi$ is the viscous dissipation function, shown later in eq. (1.45a). The quantity between parentheses on the left-hand side of eq. (1.26) is equal to zero [cf. eq. (1.6)]. In the special case where the flow can be modeled as incompressible and two-dimensional, the viscous dissipation function reduces to

$$
\Phi=2\left[\left(\frac{\partial u}{\partial x}\right)^{2}+\left(\frac{\partial v}{\partial y}\right)^{2}\right]+\left(\frac{\partial u}{\partial y}+\frac{\partial v}{\partial x}\right)^{2}
$$

To express eq. (1.26) in terms of enthalpy, we use the thermodynamics definition $h=e+(1 / \rho) P$; hence,

$$
\frac{D h}{D t}=\frac{D e}{D t}+\frac{1}{\rho} \frac{D P}{D t}-\frac{P}{\rho^{2}} \frac{D \rho}{D t}
$$

In addition, we can express the directional heat fluxes $q_{x}^{\prime \prime}$ and $q_{y}^{\prime \prime}$ in terms of the local temperature gradients; that is, we invoke the Fourier law of heat conduction, 


$$
\mathbf{q}^{\prime \prime}=-k \nabla T
$$

Then, combining eqs. (1.26), (1.28), and (1.29) in the manner desired, we obtain

$$
\rho \frac{D h}{D t}=\nabla \cdot(k \nabla T)+q^{\prime \prime \prime}+\frac{D P}{D t}+\mu \Phi-\frac{P}{\rho}\left(\frac{D \rho}{D t}+\rho \nabla \cdot \mathbf{v}\right)
$$

Finally, we learn from the mass conservation equation (1.6) that the last terms in parentheses in eq. (1.30) add up to zero; in conclusion, the first law of thermodynamics reduces to

$$
\rho \frac{D h}{D t}=\nabla \cdot(k \nabla T)+q^{\prime \prime \prime}+\frac{D P}{D t}+\mu \Phi
$$

In order to express the energy equation (1.31) in terms of temperature, it is tempting to replace the specific enthalpy on the left-hand side by the product of specific heat $X$ temperature. This move is correct only in cases where the fluid behaves like an ideal gas (see the ideal gas model, Table 1.1). In general, the change in specific enthalpy for a single-phase substance is expressed by the canonical relation for enthalpy [3],

$$
d h=T d s+\frac{1}{\rho} d P
$$

where $T$ is the absolute temperature and $d s$ the specific entropy change,

$$
d s=\left(\frac{\partial s}{\partial T}\right)_{P} d T+\left(\frac{\partial s}{\partial P}\right)_{T} d P
$$

From the last of Maxwell's relations [3, p. 173], we have

$$
\left(\frac{\partial s}{\partial P}\right)_{T}=-\left[\frac{\partial(1 / \rho)}{\partial T}\right]_{P}=\frac{1}{\rho^{2}}\left(\frac{\partial \rho}{\partial T}\right)_{P}=-\frac{\beta}{\rho}
$$

where $\beta$ is the coefficient of thermal expansion,

$$
\beta=-\frac{1}{\rho}\left(\frac{\partial \rho}{\partial T}\right)_{P}
$$

Table 1.1 also shows that 


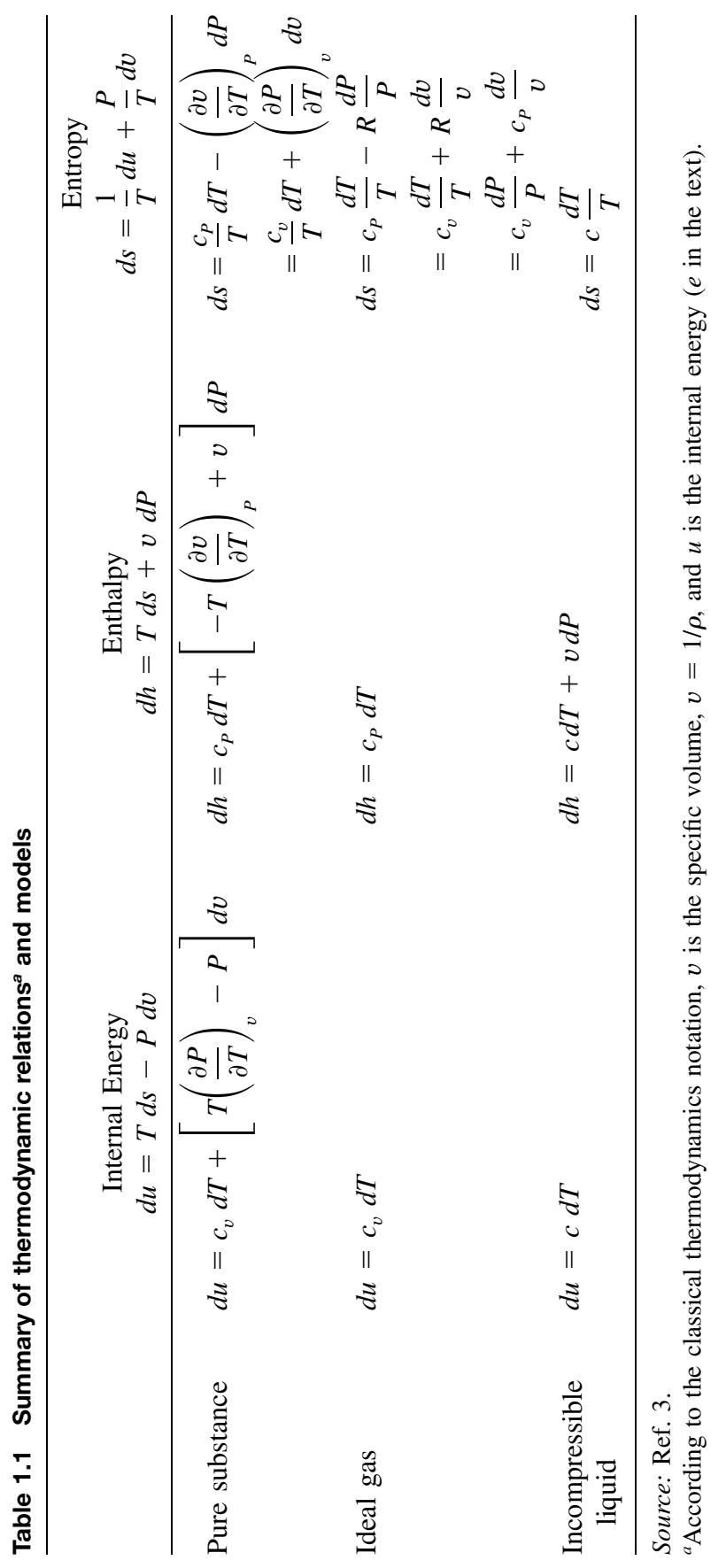




$$
\left(\frac{\partial s}{\partial T}\right)_{P}=\frac{c_{P}}{T}
$$

Together, eqs. (1.32)-(1.36) state that

$$
d h=c_{P} d T+\frac{1}{\rho}(1-\beta T) d P
$$

in other words, the left-hand side of the energy equation (1.31) is

$$
\rho \frac{D h}{D t}=\rho c_{P} \frac{D T}{D t}+(1-\beta T) \frac{D P}{D t}
$$

The "temperature" formulation of the first law of thermodynamics is therefore

$$
\rho c_{P} \frac{D T}{D t}=\nabla \cdot(k \nabla T)+q^{\prime \prime \prime}+\beta T \frac{D P}{D t}+\mu \Phi
$$

with the following special forms:

Ideal gas $(\beta=1 / T)$ :

$$
\rho c_{P} \frac{D T}{D t}=\nabla \cdot(k \nabla T)+q^{\prime \prime \prime}+\frac{D P}{D t}+\mu \Phi
$$

Incompressible liquid $(\beta=0)$ :

$$
\rho c \frac{D T}{D t}=\nabla \cdot(k \nabla T)+q^{\prime \prime \prime}+\mu \Phi
$$

Most of the convection problems addressed in this book obey an even simpler model: namely, constant fluid conductivity $k$, zero internal heat generation $q^{\prime \prime \prime}$, negligible viscous dissipation $\mu \Phi$, and negligible compressibility effect $\beta T$ $D P / D t$. The energy equation for this model is

$$
\rho c_{P} \frac{D T}{D t}=k \nabla^{2} T
$$

or, in terms of specific coordinate systems (Fig. 1.1): 
Cartesian $(x, y, z)$ :

$$
\rho c_{P}\left(\frac{\partial T}{\partial t}+u \frac{\partial T}{\partial x}+v \frac{\partial T}{\partial y}+w \frac{\partial T}{\partial z}\right)=k\left(\frac{\partial^{2} T}{\partial x^{2}}+\frac{\partial^{2} T}{\partial y^{2}}+\frac{\partial^{2} T}{\partial z^{2}}\right)
$$

Cylindrical $(r, \theta, z)$ :

$$
\begin{aligned}
\rho c_{P} & \left(\frac{\partial T}{\partial t}+v_{r} \frac{\partial T}{\partial r}+\frac{v_{\theta}}{r} \frac{\partial T}{\partial \theta}+v_{z} \frac{\partial T}{\partial z}\right) \\
=k & {\left[\frac{1}{r} \frac{\partial}{\partial r}\left(r \frac{\partial T}{\partial r}\right)+\frac{1}{r^{2}} \frac{\partial^{2} T}{\partial \theta^{2}}+\frac{\partial^{2} T}{\partial z^{2}}\right] }
\end{aligned}
$$

Spherical $(r, \phi, \theta)$ :

$$
\begin{aligned}
\rho c_{P} & \left(\frac{\partial T}{\partial t}+v_{r} \frac{\partial T}{\partial r}+\frac{v_{\phi}}{r} \frac{\partial T}{\partial \phi}+\frac{v_{\theta}}{r \sin \phi} \frac{\partial T}{\partial \theta}\right) \\
& =k\left[\frac{1}{r^{2}} \frac{\partial}{\partial r}\left(r^{2} \frac{\partial T}{\partial r}\right)+\frac{1}{r^{2} \sin \phi} \frac{\partial}{\partial \phi}\left(\sin \phi \frac{\partial T}{\partial \phi}\right)+\frac{1}{r^{2} \sin ^{2} \phi} \frac{\partial^{2} T}{\partial \theta^{2}}\right]
\end{aligned}
$$

If the fluid can be modeled thermodynamically as an incompressible liquid, then, as in eq. (1.41), the specific heat at constant pressure $c_{P}$ is replaced by the lone specific heat of the incompressible liquid, $c$ (Table 1.1).

When dealing with extremely viscous flows of the type encountered in lubrication problems or the piping of crude oil, the model above is improved by taking into account the internal heating due to viscous dissipation,

$$
\rho c_{P} \frac{D T}{D t}=k \nabla^{2} T+\mu \Phi
$$

In three dimensions, the general viscous dissipation function can be expressed as follows:

Cartesian $(x, y, z)$ :

$$
\begin{aligned}
\Phi= & 2\left[\left(\frac{\partial u}{\partial x}\right)^{2}+\left(\frac{\partial v}{\partial y}\right)^{2}+\left(\frac{\partial w}{\partial z}\right)^{2}\right] \\
& +\left[\left(\frac{\partial u}{\partial y}+\frac{\partial v}{\partial x}\right)^{2}+\left(\frac{\partial v}{\partial z}+\frac{\partial w}{\partial y}\right)^{2}+\left(\frac{\partial w}{\partial x}+\frac{\partial u}{\partial z}\right)^{2}\right] \\
& -\frac{2}{3}\left(\frac{\partial u}{\partial x}+\frac{\partial v}{\partial y}+\frac{\partial w}{\partial z}\right)^{2}
\end{aligned}
$$


Cylindrical $(r, \theta, z)$ :

$$
\begin{aligned}
\Phi= & 2\left[\left(\frac{\partial v_{r}}{\partial r}\right)^{2}+\left(\frac{1}{r} \frac{\partial v_{\theta}}{\partial \theta}+\frac{v_{r}}{r}\right)^{2}+\left(\frac{\partial v_{z}}{\partial z}\right)^{2}\right. \\
& +\frac{1}{2}\left(\frac{\partial v_{\theta}}{\partial r}-\frac{v_{\theta}}{r}+\frac{1}{r} \frac{\partial v_{r}}{\partial \theta}\right)^{2}+\frac{1}{2}\left(\frac{1}{r} \frac{\partial v_{z}}{\partial \theta}+\frac{\partial v_{\theta}}{\partial z}\right)^{2} \\
& \left.+\frac{1}{2}\left(\frac{\partial v_{r}}{\partial z}+\frac{\partial v_{z}}{\partial r}\right)^{2}-\frac{1}{3}(\nabla \cdot \mathbf{v})^{2}\right]
\end{aligned}
$$

Spherical $(r, \phi, \theta)$ :

$$
\begin{aligned}
\Phi= & 2\left\{\left[\left(\frac{\partial v_{r}}{\partial r}\right)^{2}+\left(\frac{1}{r} \frac{\partial v_{\phi}}{\partial \phi}+\frac{v_{r}}{r}\right)^{2}+\left(\frac{1}{r \sin \phi} \frac{\partial v_{\theta}}{\partial \theta}+\frac{v_{r}}{r}+\frac{v_{\phi} \cot \phi}{r}\right)^{2}\right]\right. \\
& +\frac{1}{2}\left[r \frac{\partial}{\partial r}\left(\frac{v_{\phi}}{r}\right)+\frac{1}{r} \frac{\partial v_{r}}{\partial \phi}\right]^{2}+\frac{1}{2}\left[\frac{\sin \phi}{r} \frac{\partial}{\partial \phi}\left(\frac{v_{\theta}}{r \sin \phi}\right)+\frac{1}{r \sin \phi} \frac{\partial v_{\theta}}{\partial \theta}\right]^{2} \\
& \left.+\frac{1}{2}\left[\frac{1}{r \sin \phi} \frac{\partial v_{r}}{\partial \theta}+r \frac{\partial}{\partial r}\left(\frac{v_{\theta}}{r}\right)\right]^{2}\right\}-\frac{2}{3}(\nabla \cdot \mathbf{v})^{2}
\end{aligned}
$$

If the density does not vary significantly through the flow field, $\nabla \cdot \mathbf{v}=0$ [eq. (1.6)] and the last term in each of expressions (1.45) vanishes.

It is worth reviewing the constant- $\rho$ approximation that led to eq. (1.8) and recognizing that it differs conceptually from the "incompressible substance model" of thermodynamics. The latter is considerably more restrictive than the "nearly constant" density model, eq. (1.8). For example, a compressible substance such as air can flow in such a way that eq. (1.8) is a very good approximation of eq. (1.6).

For the restrictive class of fluids that are "incompressible" from the thermodynamic point of view, the specific heat at constant pressure $c_{P}$ can be replaced by the lone specific heat of the fluid, $c$, on the left side of eq. (1.39). Water, liquid mercury, and engine oil are examples of fluids for which this substitution is justified. There are even convection problems in which the moving materials are actually solid (e.g., a roller and its substrate, in the zone of elastic contact). In such cases the $c_{P}=c$ substitution is permissible also.

It is important to note that the specific heat at constant volume $c_{v}$ does not belong on the left side of eq. (1.39). This observation is important because Fourier [5,6], and later Poisson [7], who were the first to derive the energy equation for a convective flow, wrote $c$ on the left side of eq. (1.39). They made this choice because their analyses were aimed specifically at incompressible fluids (liquids), for which $c$ happens to have nearly the same value as $c_{P}$. Because of this choice, they did not have to account for the $P d V$ type of work done by the fluid packet as it expands or contracts in the flow field. In the modern era, however, the use of $c_{v}$ instead of $c_{P}$ is an error. 
The prethermodynamics (caloric conservation) origins of the science of convection heat transfer are also responsible for the "thermal energy equation" label that some prefer to attach to eq. (1.39) without the $\beta T D P / D t$ term. This terminology is sometimes used to stress (incorrectly) the conservation of "thermal" energy as something distinct from "mechanical and thermal" energy. In classical thermodynamics, however, this distinction disappeared as soon as the first law of thermodynamics was enunciated, that is, as soon as the thermodynamic property "energy" was defined, which happened in the years 18501851 (see Ref. 3, pp. 30-32).

Equation (1.39) represents the first law of thermodynamics. This law proclaims the conservation of the sum of energy change (the property) and energy interactions (heat transfer and work transfer). The suggestion that mechanical effects (e.g., work transfer) are absent from eq. (1.39) when the $\beta T D P / D t$ term is absent is erroneous. The presence of $c_{P}$ on the left side of the equation is the sign that each fluid packet expands or contracts (i.e., it does $P d V$-type work) as it rides on the flow. The terms $q^{\prime \prime \prime}$ and $\mu \Phi$ are work transfer rate terms also.

\subsection{SECOND LAW OF THERMODYNAMICS}

Any discussion of the basic principles of convective heat transfer must include the second law of thermodynamics, not because the second law is necessary for determining the flow and temperature field (it is not, because it is not an equation), but because the second law is the basis for much of the engineering motive (objective, purpose) for formulating and solving convection problems. For example, in the development of knowhow for the heat exchanger industry, we strive for improved thermal contact (enhanced heat transfer) and reduced pump power loss in order to improve the thermodynamic efficiency of the heat exchanger. Good heat exchanger design means, ultimately, efficient thermodynamic performance, that is, minimum generation of entropy or minimum destruction of exergy in the power/refrigeration system incorporating the heat exchanger [8-10]. For this reason, it is useful to review the second law and in this way to explain the commonsense origin of the engineering questions that led to today's field of convective heat transfer.

The second law of thermodynamics states that all real-life processes are irreversible: In the case of a control volume, as in Fig. 1.1, this statement is

$$
\frac{\partial S_{\mathrm{cv}}}{\partial t} \geq \sum \frac{q_{i}}{T_{i}}+\sum_{\substack{\text { inlet } \\ \text { ports }}} \dot{m} s-\sum_{\substack{\text { outlet } \\ \text { ports }}} \dot{m} s
$$

where $S_{\mathrm{cv}}$ is the instantaneous entropy inventory of the control volume, $\dot{m} s$ represents the entropy flows (streams) into and out of the control volume, and $T_{i}$ is the absolute temperature of the boundary crossed by the heat transfer 
interaction $q_{i} *$ The irreversibility of the process is measured by the strength of the inequality sign in eq. (1.46), or by the entropy generation rate $S_{\text {gen }}$, defined as

$$
S_{\text {gen }}=\frac{\partial S_{\mathrm{cv}}}{\partial t}-\sum \frac{q_{i}}{T_{i}}-\sum_{\substack{\text { inlet } \\ \text { ports }}} \dot{m} s+\sum_{\substack{\text { outlet } \\ \text { ports }}} \dot{m} s \geq 0
$$

It is easy to show that the rate of destruction of useful work in an engineering system, $W_{\text {lost }}$, is directly proportional to the rate of entropy generation [8-10],

$$
W_{\text {lost }}=T_{0} S_{\text {gen }}
$$

where $T_{0}$ is the absolute temperature of the ambient temperature reservoir $\left(T_{0}\right.$ $=$ constant). Equation (1.48) stresses the engineering importance of estimating the irreversibility or entropy generation rate of convective heat transfer processes: If not used wisely, these processes contribute to the waste of precious fuel resources.

Based on an analysis similar to the analyses presented for mass conservation, force balances, and the first law of thermodynamics, the second law (1.47) may be applied to a finite-size control volume $\Delta x \Delta y \Delta z$ at an arbitrary point $(x, y, z)$ in a flow field. Thus, the rate of entropy generation per unit time and per unit volume $S_{\text {gen }}^{\prime \prime \prime}$ is $[8,9]$

$$
S_{\text {gen }}^{\prime \prime \prime}=\underbrace{\frac{k}{T^{2}}(\nabla T)^{2}}_{\geq 0}+\underbrace{\frac{\mu}{T} \Phi}_{\geq 0} \geq 0
$$

where $k$ and $\mu$ are assumed constant. In a two-dimensional convection situation such as in Figs. 1.1-1.3, the local entropy generation rate (1.49) yields

$$
\begin{aligned}
S_{\text {gen }}^{\prime \prime \prime}= & \frac{k}{T^{2}}\left[\left(\frac{\partial T}{\partial x}\right)^{2}+\left(\frac{\partial T}{\partial y}\right)^{2}\right] \\
& +\frac{\mu}{T}\left\{2\left[\left(\frac{\partial u}{\partial x}\right)^{2}+\left(\frac{\partial v}{\partial y}\right)^{2}\right]+\left(\frac{\partial u}{\partial y}+\frac{\partial v}{\partial x}\right)^{2}\right\} \geq 0
\end{aligned}
$$

In the last two equations, $T$ represents the absolute temperature of the point where $S_{\text {gen }}^{\prime \prime \prime}$ is being evaluated. The two-dimensional expression (1.50) illustrates the competition between viscous dissipation and imperfect thermal contact (finite-temperature gradients) in the generation of entropy via convective heat 
transfer. The two-sided character of entropy generation in convective heat transfer was illustrated most recently by Mahmud and Fraser [11].

Equations (1.48) and (1.50) constitute the bridge between two research activities: fundamental convection heat transfer and applied thermodynamics (entropy generation minimization). Beginning with Chapter 2, we focus on the fundamental problems of determining the flow and temperature fields in a given convection heat transfer configuration. However, through eq. (1.50), we are invited to keep in mind that these fields contribute hand-in-hand to downgrading the thermodynamic merit of the engineering device that ultimately employs the convection process under consideration. The science of adjusting the convection process so that it destroys the least exergy (subject to various system constraints) is the focus of entropy generation minimization; this activity has been reviewed in Refs. 8-10. The generation of flow configuration (geometry, architecture) for maximal performance under constraints is constructal theory and design [12-15].

\subsection{RULES OF SCALE ANALYSIS}

This section is designed to familiarize the student with the commonsense problem-solving method of scale analysis or scaling. This section is necessary because scale analysis is used extensively throughout the book; in fact, scale analysis is recommended as the premier method for obtaining the most information per unit of intellectual effort. Furthermore, this section is necessary because scale analysis is not discussed in the heat transfer and fluid mechanics textbooks of our time, despite the fact that it is a precondition for good analysis in dimensionless form. Scale analysis is often confused with dimensional analysis or the often arbitrary nondimensionalization of the governing equations before performing a perturbation analysis or a numerical simulation on the computer.

The object of scale analysis is to use the basic principles of convective heat transfer to produce order-of-magnitude estimates for the quantities of interest. This means that if one of the quantities of interest is the thickness of the boundary layer in forced convection, the object of scale analysis is to determine whether the boundary layer thickness is measured in millimeters or meters. Note that scale analysis goes beyond dimensional analysis (whose objective is to determine the dimension of boundary layer thickness, namely, length). When done properly, scale analysis anticipates within a factor of order one (or within percentage points) the expensive results produced by "exact" analyses. The value of scale analysis is remarkable, particularly when we realize that the notion of "exact analysis" is as false and ephemeral as the notion of "experimental fact."

As the first example of scale analysis, consider a problem from the field of conduction heat transfer [16]. In Fig. 1.4 we see a plate plunged at $t=0$ into a highly conducting fluid, such that the surfaces of the plate instantaneously 


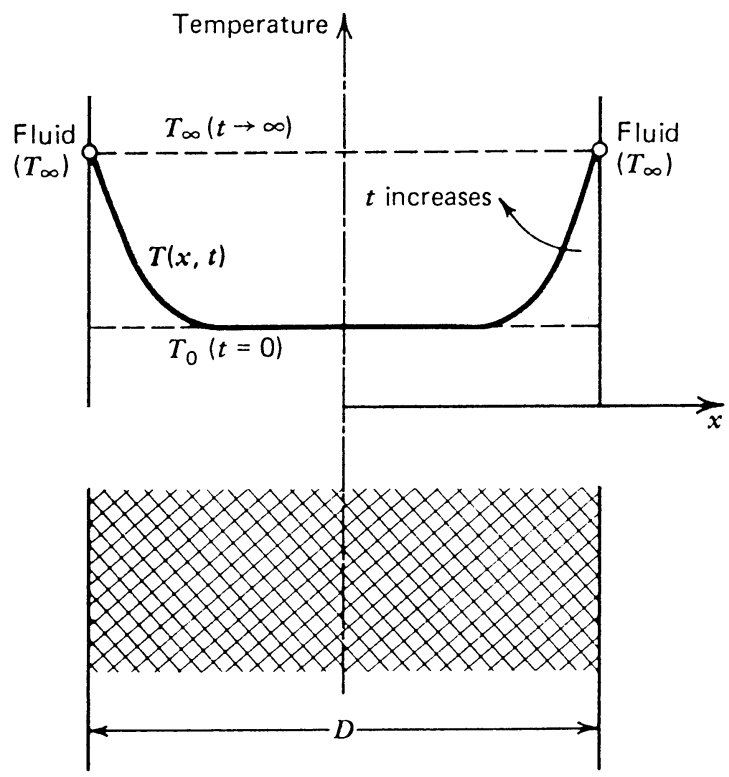

Figure 1.4 Transient heat conduction in a slab with sudden temperature change on the boundaries.

assume the fluid temperature $T_{\infty}=T_{0}+\Delta T$. Suppose that we are interested in estimating the time needed by the thermal front to penetrate the plate, that is, the time until the center plane of the plate "feels" the heating imposed on the outer surfaces.

To answer the question above, we focus on a half-plate of thickness $D / 2$ and the energy equation for pure conduction in one direction:

$$
\rho c_{P} \frac{\partial T}{\partial t}=k \frac{\partial^{2} T}{\partial x^{2}}
$$

Next, we estimate the order of magnitude of each of the terms appearing in eq. (1.51). On the left-hand side we have

$$
\rho c_{P} \frac{\partial T}{\partial t} \sim \rho c_{P} \frac{\Delta T}{t}
$$

In other words, the scale of the temperature change (in the chosen space and in a time of order $t$ ) is $\Delta T$. On the right-hand side we obtain

$$
k \frac{\partial^{2} T}{\partial x^{2}}=k \frac{\partial}{\partial x}\left(\frac{\partial T}{\partial x}\right) \sim \frac{k}{D / 2} \frac{\Delta T}{D / 2}=\frac{k \Delta T}{(D / 2)^{2}}
$$


Equating the two orders of magnitude (1.52) and (1.53), as required by the energy equation (1.51), we find the answer to the problem

$$
t \sim \frac{(D / 2)^{2}}{\alpha}
$$

where $\alpha$ is the thermal diffusivity of the medium, $k / \rho c_{P}$. The penetration time (1.54) compares well with any order-of-magnitude interpretation of the exact solution to this classical problem [16]. However, the time and effort associated with deriving eq. (1.54) do not compare with the labor required by Fourier analysis and the graphical presentation of Fourier series.

Based on this introductory example, the following rules of scale analysis are worth stressing.

- Rule 1. Always define the spatial extent of the region in which you perform the scale analysis. In the example of Fig. 1.4, the size of the region of interest is $D / 2$. In other problems, such as boundary layer flow, the size of the region of interest is unknown; as shown in Chapter 2, the scale analysis begins by selecting the region and by labeling the unknown thickness of this region $\delta$. Any scale analysis of a flow or a flow region that is not uniquely defined is pure nonsense.

- Rule 2. One equation constitutes an equivalence between the scales of two dominant terms appearing in the equation. In the transient conduction example of Fig. 1.4, the left-hand side of eq. (1.51) could only be of the same order of magnitude as the right-hand side. The two terms appearing in eq. (1.51) are the dominant terms (considering that the discussion referred to pure conduction); in general, the energy equation can contain many more terms [eq. (1.39)], not all of them important. The reasoning for selecting the dominant scales from many scales is condensed in rules 3-5.

- Rule 3. If in the sum of two terms,

$$
c=a+b
$$

the order of magnitude of one term is greater than the order of magnitude of the other term,

$$
O(a)>O(b)
$$

then the order of magnitude of the sum is dictated by the dominant term:

$$
O(c)=O(a)
$$

The same conclusion holds if instead of eq. (1.55), we have the difference $c=a-b$ or $c=-a+b$. 
- Rule 4. If in the sum of two terms,

$$
c=a+b
$$

the two terms are of the same order of magnitude,

$$
O(a)=O(b)
$$

then the sum is also of the same order of magnitude:

$$
O(c) \sim O(a) \sim O(b)
$$

- Rule 5. In any product

$$
p=a b
$$

the order of magnitude of the product is equal to the product of the orders of magnitude of the two factors

$$
O(p)=O(a) O(b)
$$

If, instead of eq. (1.60), we have the ratio

$$
r=\frac{a}{b}
$$

then

$$
O(r)=\frac{O(a)}{O(b)}
$$

In addition to having its own set of rules, scale analysis requires special care with regard to notation. In rules $1-5$, we used the following symbols:

$\sim \quad$ is of the same order of magnitude as

$O(a)$ the order of magnitude of $a$

$>\quad$ greater than, in an order-of-magnitude sense

For brevity, the scale analyses included in this book employ the language of expressions (1.56), (1.57), (1.61), and (1.63) without the repetitive potentially confusing notation $O(\cdot)$ for "order of magnitude."

Scale analysis is now employed widely in heat transfer, and the fundamental scaling results that have been developed go beyond the first steps presented in 
this book. For example, Bhattacharjee and Grosshandler [17] have reported the scale analysis of a pressure-driven wall jet. Li and Djilali [18] have used scale analysis to describe the behavior of separating flows behind backward-facing steps (separation bubbles). Li [19] has reported the scaling results for jet diffusion flames.

\subsection{HEATLINES FOR VISUALIZING CONVECTION}

The opportunity to actually "see" the solution to a problem is essential to a problem solver's ability to learn from experience and in this way to improve his or her technique. In convection problems it is important to visualize the flow of fluid and, riding on this, the flow of energy. For example, in the twodimensional Cartesian configuration of Fig. 1.1, it has been common practice to define a streamfunction $\psi(x, y)$ as

$$
u=\frac{\partial \psi}{\partial y}, \quad v=-\frac{\partial \psi}{\partial x}
$$

such that the mass continuity equation for incompressible flow,

$$
\frac{\partial u}{\partial x}+\frac{\partial v}{\partial y}=0
$$

is satisfied identically. It is easy to verify that the actual flow is locally parallel to the $\psi=$ constant line passing through the point of interest. Therefore, although there are no substitutes for $u$ and $v$ as bearers of precise information regarding the local flow, the family of $\psi=$ constant streamlines provides a much needed bird's-eye view of the entire flow field and its main characteristics.

In convection, the transport of energy through the flow field is a combination of both thermal diffusion and enthalpy flow [cf. eq. (1.42)]. For any such field, Kimura and Bejan [20] defined a new function $H(x, y)$ such that the net flow of energy (thermal diffusion and enthalpy flow) is zero across each $H=$ constant line. The mathematical definition of the heatfunction $H$ follows in the steps of eqs. (1.64) if this time the aim is to satisfy the energy equation. For steady-state two-dimensional convection through a constant-property homogeneous fluid, eq. (1.42) becomes

$$
u \frac{\partial T}{\partial x}+v \frac{\partial T}{\partial y}=\alpha\left(\frac{\partial^{2} T}{\partial x^{2}}+\frac{\partial^{2} T}{\partial y^{2}}\right)
$$




$$
\frac{\partial}{\partial x}\left(\rho c_{P} u T-k \frac{\partial T}{\partial x}\right)+\frac{\partial}{\partial y}\left(\rho c_{P} v T-k \frac{\partial T}{\partial y}\right)=0
$$

The heatfunction is defined as follows:

Net energy flow in the $x$ direction:

$$
\frac{\partial H}{\partial y}=\rho c_{P} u\left(T-T_{\mathrm{ref}}\right)-k \frac{\partial T}{\partial x}
$$

Net energy flow in the y direction:

$$
-\frac{\partial H}{\partial x}=\rho c_{P} v\left(T-T_{\text {ref }}\right)-k \frac{\partial T}{\partial y}
$$

so that the heatfunction $H(x, y)$ satisfies eq. (1.66) identically. Note that the definition above also applies to convection through a fluid-saturated porous medium, where eq. (1.66) accounts for energy conservation.

The reference temperature $T_{\text {ref }}$ is, in principle, an arbitrary constant that can be selected based on convention. Patterns of $H=$ constant heatlines are instructive when $T_{\text {ref }}$ is the lowest temperature that occurs in the heat transfer configuration. For example, if the wall shown in Fig. 2.1 is warmer than the free stream, $T_{0}>T_{\infty}$, the choice of reference temperature is $T_{\text {ref }}=T_{\infty}$. For a meaningful comparison of the heatlines of one flow with the heatlines of another flow, I propose that $T_{\text {ref }}$ always be set equal to the lowest temperature of the flow field.

If the fluid flow subsides $(u=v=0)$, the heatlines become identical to the heat flux lines employed frequently in the study of conduction phenomena. Therefore, as a heat transfer visualization technique, the use of heatlines is the convection counterpart or generalization of a standard technique (heat flux lines) used in conduction. It is also interesting to point out that the contemporary use of $T=$ constant lines is not a proper way to visualize heat transfer in the field of convection; isotherms are a proper heat transfer visualization tool only in the field of conduction (where, in fact, they have been invented) because only there are they locally orthogonal to the true direction of energy flow. The use of $T=$ constant lines to visualize convection heat transfer makes as little sense as using $P=$ constant lines to visualize fluid flow.

The heatline method for the visualization of convective heat transfer was proposed in the first edition of this book (1984), along with a first application to natural convection in an enclosure heated from the side [20]. The method has since been adopted and extended in several ways in the post-1984 heat transfer literature [21-43]. 


\section{REFERENCES}

1. D. B. Guralnik, ed., Webster's New World Dictionary, 2nd college ed., World Publishing Company, New York, 1970, p. 310.

2. D. P. Simpson, Cassell's Latin Dictionary, Macmillan, New York, 1978, p. 150.

3. A. Bejan, Advanced Engineering Thermodynamics, 2nd ed., Wiley, New York, 1997, p. 193.

4. W. M. Rohsenow and H. Y. Choi, Heat, Mass and Momentum Transfer, PrenticeHall, Englewood Cliffs, NJ, 1961, p. 48.

5. J. B. J. Fourier, Mémoire d'analyse sur le mouvement de la chaleur dans les fluides, in Mémoires de l'Académie Royale des Sciences de l'Institut de France, Didot, Paris, 1833, pp. 507-530 (presented on Sept. 4, 1820).

6. J. B. J. Fourier, Oeuvres de Fourier, G. Darboux, ed., Vol. 2, Gauthier-Villars, Paris, 1890, pp. 595-614.

7. S. D. Poisson, Théorie Mathématique de la Chaleur, Paris, 1835, Chapter 4, p. 86.

8. A. Bejan, Entropy Generation through Heat and Fluid Flow, Wiley, New York, 1982.

9. A. Bejan, Entropy Generation Minimization, CRC Press, Boca Raton, FL, 1996.

10. A. Bejan, G. Tsatsaronis, and M. Moran, Thermal Design and Optimization, Wiley, New York, 1996.

11. S. Mahmud and R. A. Fraser, The second law analysis in fundamental convective heat transfer problems, Int. J. Therm. Sci., Vol. 42, 2003, pp. 177-186.

12. A. Bejan, Shape and Structure, from Engineering to Nature, Cambridge University Press, Cambridge, 2000.

13. R. N. Rosa, A. H. Reis, and A. F. Miguel, eds., Bejan's Constructal Theory of Shape and Structure, Évora Geophysics Center, University of Évora, Portugal, 2004.

14. H. Poirier, Une théorie explique l'intelligence de la nature, Science \& Vie, No. 1034, November 2003, pp. 44-63.

15. M. Torre, La Natura, vi svelo le formule della perfezione, La Macchina del Tempo, Nos. 1-2, Year 5, January-February 2004, pp. 36-46.

16. A. Bejan, Heat Transfer, Wiley, New York, 1993, Chapter 4.

17. S. Bhattacharjee and W. L. Grosshandler, The formation of a wall jet near a high temperature wall under microgravity environment, ASME HTD, Vol. 96, 1988, pp. 711-716.

18. X. Li and N. Djilali, On the scaling of separation bubbles, JSME Int. J., Ser. B, Vol. 38, No. 4, 1995, pp. 541-548.

19. X. Li, On the scaling of the visible lengths of jet diffusion flames, J. Energy Resour. Technol., Vol. 118, 1996, pp. 128-133.

20. S. Kimura and A. Bejan, The "heatline" visualization of convective heat transfer, J. Heat Transfer, Vol. 105, 1983, pp. 916-919.

21. D. Littlefield and P. Desai, Buoyant laminar convection in a vertical cylindrical annulus, J. Heat Transfer, Vol. 108, 1986, pp. 814-821.

22. O. V. Trevisan and A. Bejan, Combined heat and mass transfer by natural convection in a vertical enclosure, J. Heat Transfer, Vol. 109, 1987, pp. 104-109. 
23. F. L. Bello-Ochende, Analysis of heat transfer by free convection in tilted rectangular cavities using the energy analogue of the stream function, Int. J. Mech. Eng. Ed., Vol. 15, 1987, pp. 91-98.

24. F. L. Bello-Ochende, A heat function formulation for thermal convection in a square cavity, Int. Comm. Heat Mass Transfer, Vol. 15, 1988, pp. 193-202.

25. A. M. Morega, The heat function approach to the thermo-magnetic convection of electroconductive melts, Rev. Roum. Sci. Tech. Ser. Electrotech. Energ., Vol. 33, 1988, pp. 33-39.

26. S. K. Aggarwal and A. Manhapra, Use of heatlines for unsteady buoyancy-driven flow in a cylindrical enclosure, J. Heat Transfer, Vol. 111, 1989, pp. 576-578.

27. S. K. Aggarwal and A. Manhapra, Transient natural convection in a cylindrical enclosure nonuniformly heated at the top wall, Numer. Heat Transfer, Part A, Vol. 15, 1989, pp. 341-356.

28. C. J. Ho, Y. H. Lin, and T. C. Chen, A numerical study of natural convection in concentric and eccentric horizontal cylindrical annuli with mixed boundary conditions, Int. J. Heat Fluid Flow, Vol. 10, 1989, pp. 40-47.

29. C. J. Ho and Y. H. Lin, Thermal convection heat transfer of air/water layers enclosed in horizontal annuli with mixed boundary conditions, Wärme Stoffübertrag., Vol. 24, 1989, pp. 211-224.

30. C. J. Ho and Y. H. Lin, Natural convection of cold water in a vertical annulus with constant heat flux on the inner wall, J. Heat Transfer, Vol. 112, 1990, pp. 117123.

31. A. M. Morega and A. Bejan, Heatline visualization of forced convection boundary layers, Int. J. Heat Mass Transfer, Vol. 36, 1993, pp. 3957-3966.

32. A. M. Morega and A. Bejan, Heatline visualization of forced convection in porous media, Int. J. Heat Fluid Flow, Vol. 15, 1994, pp. 42-47.

33. V. A. F. Costa, Double diffusive natural convection in a square enclosure with heat and mass diffusive walls, Int. J. Heat Mass Transfer, Vol. 40, 1997, pp. 4061-4071.

34. V. A. F. Costa, Double diffusive natural convection in enclosures with heat and mass diffusive walls, in G. De Vahl Davis and E. Leonardi. eds., Proceedings of the International Symposium on Advances in Computational Heat Transfer (CHT'97), Begell House, New York, 1998, pp. 338-344.

35. H. Y. Wang, F. Penot, and J. B. Saulnier, Numerical study of a buoyancy-induced flow along a vertical plate with discretely heated integrated circuit packages, Int. J. Heat Mass Transfer, Vol. 40, 1997, pp. 1509-1520.

36. V. A. F. Costa, Unification of the streamline, heatline and massline methods for the visualization of two-dimensional transport phenomena, Int. J. Heat Mass Transfer, Vol. 42, 1999, pp. 27-33.

37. S. J. Kim and S. P. Jang, Experimental and numerical analysis of heat transfer phenomena in a sensor tube of a mass flow controller, Int. J. Heat Mass Transfer, Vol. 44, 2001, pp. 1711-1724.

38. Q.-H. Deng and G.-F. Tang, Numerical visualization of mass and heat transport for conjugate natural convection/heat conduction by streamline and heatline, Int. J. Heat Mass Transfer, Vol. 45, 2002, pp. 2375-2385.

39. Q.-H. Deng and G.-F. Tang, Numerical visualization of mass and heat transport for mixed convective heat transfer by streamline and heatline, Int. J. Heat Mass Transfer, Vol. 45, 2002, pp. 2387-2396. 
40. A. Mukhopadhyay, X. Qin, S. K. Aggarwal, and I. K. Puri, On extension of "heatline" and "massline" concepts to reacting flows through the use of conserved scalars, J. Heat Transfer, Vol. 124, 2002, pp. 791-799.

41. V. A. F. Costa, Comment on the paper by Qi-Hong Deng and Guang-Fa Tang, Numerical visualization of mass and heat transport for conjugate natural convection/ heat conduction by streamline and heatline, Int. J. Heat Mass Transfer, Vol. 46, 2003, pp. 185-187.

42. A. F. Costa, Unified streamline, heatline and massline methods for the visualization of two-dimensional heat and mass transfer in anisotropic media, Int. J. Heat Mass Transfer, Vol. 46, 2003, pp. 1309-1320.

43. A. Mukhopadhyay, X. Qin, I. K. Puri, and S. K. Aggarwal, Visualization of scalar transport in nonreacting and reacting jets through a unified "heatline" and "massline" formulation, Numerical Heat Transfer, Part A, Vol. 44, 2003, pp. 683-704.

\section{PROBLEMS}

1.1. Consider the unsteady mass conservation equation (1.5) as it might describe the flow accelerating through a duct with a variable cross section. If the largest velocity gradient measured locally is $d u / d x$ and the largest density gradient is $d \rho / d x$, what order-of-magnitude relationship must exist between $d u / d x$ and $d \rho / d x$ for the simplified equation (1.8) to be applicable?

1.2. Derive the mass conservation equation in cylindrical coordinates [eq. (1.9)] by applying the general principle (1.1) to an elementary control volume of size $\Delta r(r \Delta \theta) \Delta z$ in Fig. $1.1 b$ (assume that $\rho=$ constant).

1.3. Derive the mass conservation statement for spherical coordinates [eq. (1.10)] by writing eq. (1.1) for the elementary control volume $(\Delta r)(r$ sin $\phi \Delta \theta)(r \Delta \phi)$ around point $(r, \theta, \phi)$ in Fig. 1.1c (assume that $\rho=$ constant).

1.4. Consider a flow in which $\rho$ and $\mu$ may be regarded as constant. Show that the $x$ momentum equation (1.18) follows from eq. (1.17) through proper use of the mass conservation principle.

1.5. Imagine a flow described by eqs. (1.9) and (1.21) in cylindrical coordinates. If the flow is situated on one side of and infinitely far from the $r=0$ origin of the coordinate system, the local three-directional increments $\Delta r, r \Delta \theta, \Delta z$ become analogous to three Cartesian increments $\Delta x, \Delta y, \Delta z$ measured away from the local point $(r, \theta, z)$ in the flow field. Show that in the limit $r \rightarrow \infty$, the transformation $\Delta r \rightarrow \Delta x, r \Delta \theta \rightarrow \Delta y$, $\Delta z \rightarrow \Delta z$ leads to the collapse of eqs. (1.9) and (1.21) into their $(x, y, z)$ Cartesian equivalents [eqs. (1.8) and (1.19)].

1.6. Consider the conservation of mass and the three force balances in spherical coordinates [eqs. (1.10) and (1.22)]. If the flow described by these 
equations is situated infinitely far from the $r=0$ origin of the spherical system, the following transformation is applicable (Fig. 1.1): $\Delta r \rightarrow \Delta x$, $r \sin \phi \Delta \theta \rightarrow \Delta y, r \Delta \phi \rightarrow \Delta z$. Show that through this transformation, in the limit $r \rightarrow \infty$, eqs. (1.10) and (1.22) become the same as eqs. (1.8) and (1.19).

1.7. Implicit in the derivation of the energy equation (1.39) is the assumption that changes in kinetic energy $V^{2} / 2$ are negligible relative to changes in internal energy $e$ [see expressions (1.25), where $e$ should, in general, be replaced by $e+V^{2} / 2$ ]. Retrace the path leading to eq. (1.39) by taking into account changes in kinetic energy; show that the result of this more rigorous analysis is identical to eq. (1.39).

1.8. Demonstrate that lost work is always proportional to entropy generation [eq. (1.48)], where $W_{\text {lost }}=W_{\text {maximum }}-W_{\text {actual }}$, and where $W_{\text {maximum }}$ corresponds to the reversible limit $\left(S_{\text {gen }}=0\right)$. Write the first law of thermodynamics for a control volume, first for the actual (real) process and then for the reversible process. Then use the definition of $W_{\text {lost }}$ and $S_{\text {gen }}$ to prove eq. (1.48).

1.9. Derive the formula for the local rate of entropy generation [eq. (1.49)]. Begin with translating the general statement (1.47) into the language of the two-dimensional control volume $\Delta x \Delta y$. Combine the resulting expression with the first law of thermodynamics as given by eq. (1.26), plus the canonical relation for internal energy (Table 1.1).

1.10. Consider the Couette flow between two parallel plates separated by a gap of width $D$ and moving relative to one another with a speed $U$. The temperature difference $\Delta T$ is imposed between the two plates. Estimate the rate of entropy generation per unit volume in this flow. What relationship must exist between $D, U, \Delta T$, and the fluid properties $\mu$ and $k$ for $S_{\text {gen }}^{\prime \prime \prime}$ to be dominated by the irreversibility due to fluid friction?

1.11. According to the one-dimensional (longitudinal) conduction model of a fin, the temperature distribution along the fin, $T(x)$, obeys the energy equation [16]

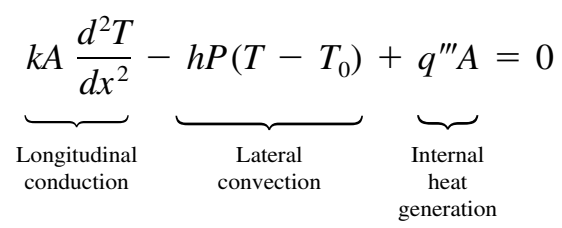

where $A, h, P$, and $q^{\prime \prime \prime}$ are the fin cross-sectional area, fin-fluid heat transfer coefficient, perimeter of the fin cross section (called the wetted perimeter), and volumetric rate of heat generation. Consider the semiinfinite fin that as shown in Fig. P1.11, is bathed by a fluid of temperature $T_{0}$ and is attached to a solid wall of temperature $T_{0}$. The heat generated by the fin is absorbed by either the fluid or the solid wall. 


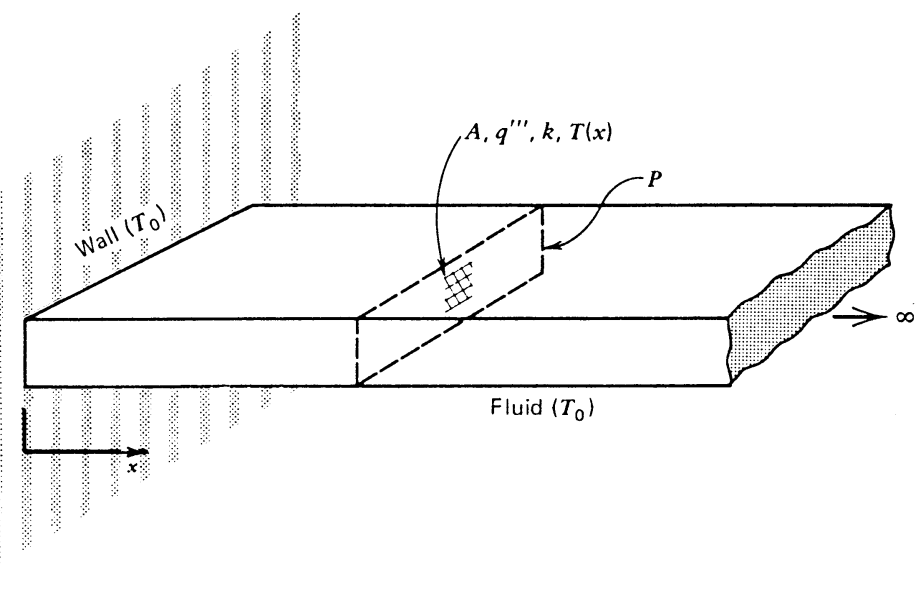

Figure P1.11

(a) As a system for scale analysis, select the fin section of length $x$, where $x$ is measured away from the wall. Let $T_{\infty}$ be the fin temperature sufficiently far from the wall. Show that if $x$ is large enough, the longitudinal conduction term becomes negligible in the energy equation.

(b) Invoking the balance between lateral convection and internal heat generation, determine the fin temperature sufficiently far from the wall, $T_{\infty}$.

(c) Determine the fin section of length $\delta$ near the wall where the heat transfer is ruled by the balance between longitudinal conduction and internal heat generation.

(d) Determine the heat transfer rate into the wall through the base of the fin.

1.12. Consider the laminar flow near a flat, solid wall, as illustrated in Fig. 2.1. The momentum equation for this flow involves the competition among three effects: inertia, pressure gradient, and friction [see eq. (2.26)]. For the purpose of scale analysis, consider a flow region of length $L$ and thickness $L$. Show that in this region, the ratio of inertia to friction is of order $\operatorname{Re}_{L}$, where $\operatorname{Re}_{L}$ is the Reynolds number based on wall length. Note that the region selected for analysis is not the boundary layer region discussed in Chapter 2. In a certain flow, the value of $\operatorname{Re}_{L}$ is $10^{3}$. What force balance rules the $L \times L$ region: inertia $\sim$ pressure, inertia $\sim$ friction, or pressure $\sim$ friction? 